\title{
The advantages and limitations of echocardiography in a unique presentation of double-sided endocarditis from intravenous drug use
}

\author{
Ben Alencherry*1, Claire Sullivan², Brian Hoit $^{3}$ \\ ${ }^{1}$ Department of Medicine, University Hospitals Case Medical Center, Cleveland, OH, USA \\ ${ }^{2}$ Harrington Heart and Vascular Institute, University Hospital Case Medical Center and Department of Medicine, Case Western \\ Reserve University, Cleveland, $\mathrm{OH}$, USA \\ ${ }^{3}$ Case Western Reserve University, Cleveland, OH, USA
}

Received: April 10, 2016

DOI: $10.5430 /$ crim.v3n3p26
Accepted: May 24, 2016

Online Published: June 12, 2016

\begin{abstract}
A 25-year-old female intravenous drug user presented with a three-week-history of fever and myalgias and was found to have infective endocarditis affecting the mitral and tricuspid valves. Blood cultures grew methicillin sensitive Staphylococcus aureus. Computed tomography showed emboli in multiple organs. Transthoracic echocardiogram revealed large vegetations on the mitral valve with moderate valvular regurgitation and a transesophageal echocardiogram identified a posterior mitral leaflet perforation. The patient underwent urgent surgical intervention after developing respiratory distress from worsening congestive heart failure. Intraoperative findings in addition to those found on echocardiography included a large abscess in the posterior wall of the left atrium. The patient underwent mitral and tricuspid valve replacement due to valve damage. This case shows a unique presentation of valvular involvement of infective endocarditis and the capabilities and limitations of echocardiography in assessing the extent of disease in this condition.
\end{abstract}

Key Words: Endocarditis, Valve perforation, Echocardiography, Mural abscess, Intravenous drug use

\section{BACKGROUND}

Infective Endocarditis (IE) is a challenging diagnosis defined by infection of a heart valve, endocardial surface, or indwelling cardiac device. ${ }^{[1]}$ Risk factors have shifted from rheumatic heart disease to intravenous drug use (IVDU), degenerative valve disease, congenital heart disease, and an increase in the number of indwelling cardiac devices and central venous lines. ${ }^{[1]}$ Complications of IE play an important role in treatment strategies including duration of antibiotic therapy and the timing of surgery, if necessary. Although not
$100 \%$ sensitive or specific, imaging including transthoracic echocardiogram (TTE) and transesophageal echocardiogram (TEE) have a central importance in the diagnosis of IE and identification of complications. ${ }^{[2]}$ This case report highlights the strengths and limitations of echocardiography in evaluation of endocarditis.

\section{Clinical presentation}

A 25-year-old female intravenous drug user was transferred to University Hospitals Case Medical Center with a three-

\footnotetext{
*Correspondence: Ben Alencherry, MD; Email: Ben.Alencherry@UHhospitals.org; Address: Department of Medicine, University Hospitals Case Medical Center, Cleveland, OH, USA.
} 
week history of fever, myalgias, productive cough, and one episode of amaurosis fugax within the past week. Vital signs upon transfer included temperature $98.4^{\circ} \mathrm{F}$, heart rate 188 beats/minute, respiratory rate 32 breaths/minute, blood pressure $103 / 58 \mathrm{mmHg}$, and oxygen saturation $100 \%$ on $40 \%$ ventimask. Physical exam was significant for altered mentation, icteric sclera, no murmurs, no signs of congestive heart failure, and no evidence of embolic or immunologic phenomena of IE. Pertinent labs demonstrated leukocytosis with left shift, transaminitis, and blood cultures growing methicillin sensitive Staphylococcus aureus. TTE revealed preserved left ventricular systolic function, a large mitral valve vegetation measuring $24 \mathrm{~mm} \times 13 \mathrm{~mm}$ on the posterior leaflet with moderate regurgitation, a $13 \mathrm{~mm} \times 8 \mathrm{~mm}$ tricuspid valve vegetation with mild to moderate regurgitation, and structurally normal aortic and pulmonic valves (see Figure $1)$.

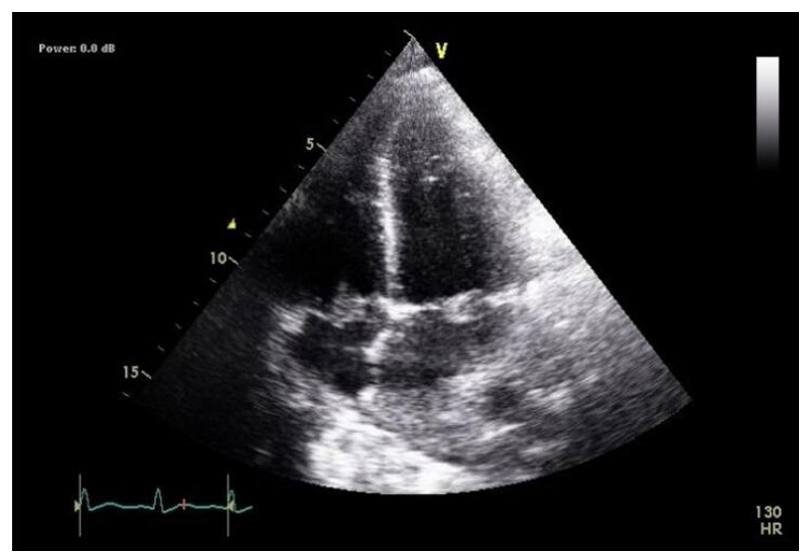

Figure 1. TTE apical four chamber image demonstrating tricuspid and mitral valve vegetations

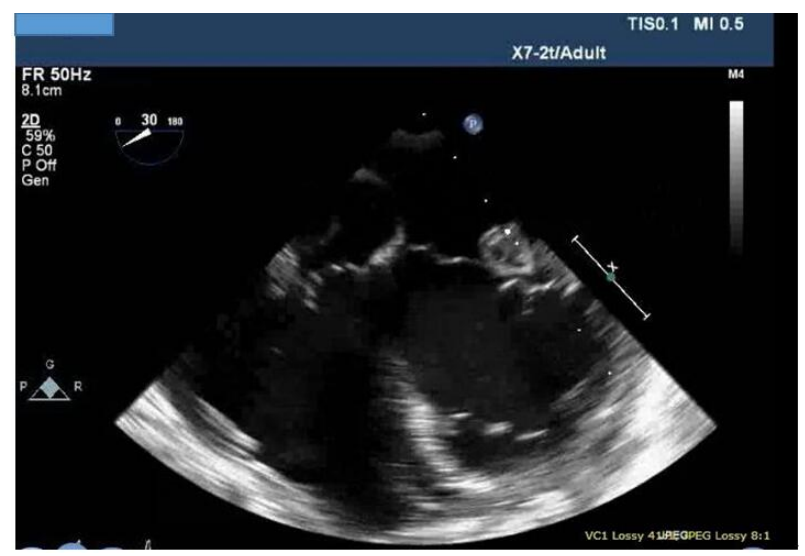

Figure 2. TEE showing large mitral valve vegetation and smaller tricuspid valve vegetation

Published by Sciedu Press

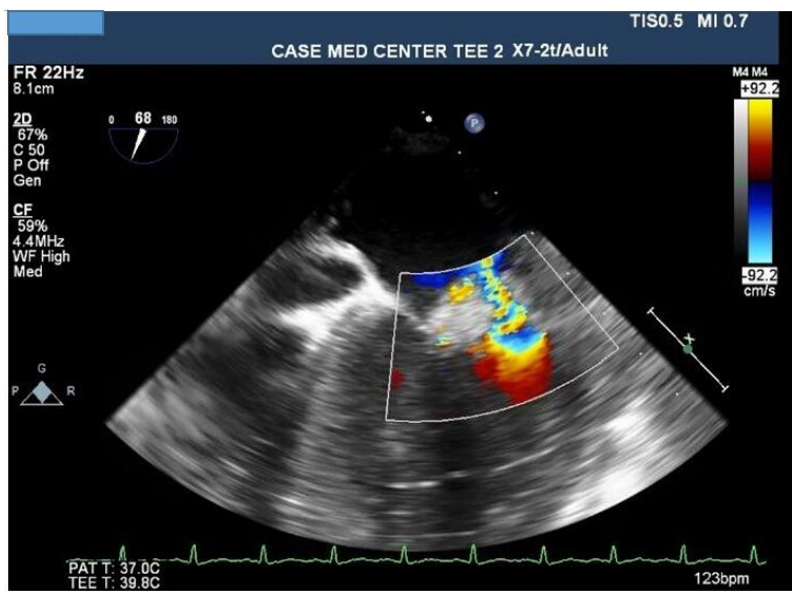

Figure 3. TEE with color Doppler demonstrating posterior mitral valve leaflet perforation

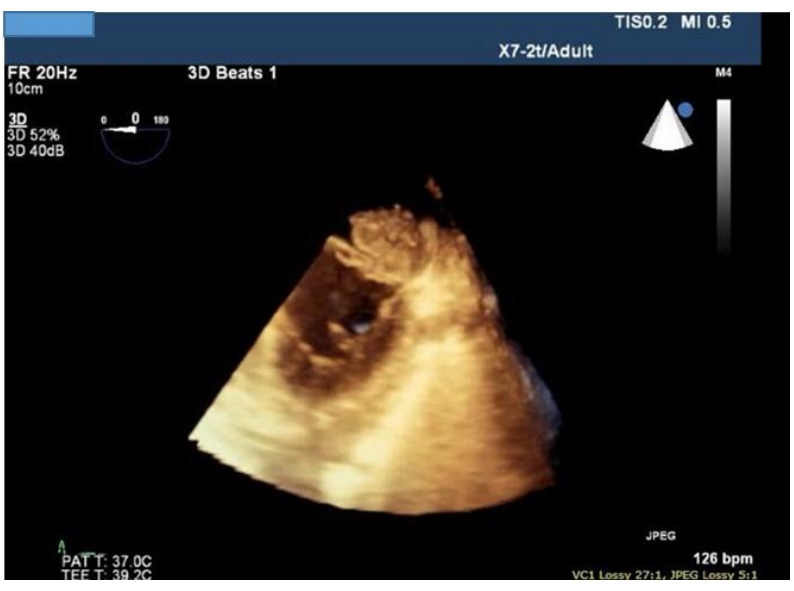

Figure 4. 3D TEE image highlighting mitral valve vegetation

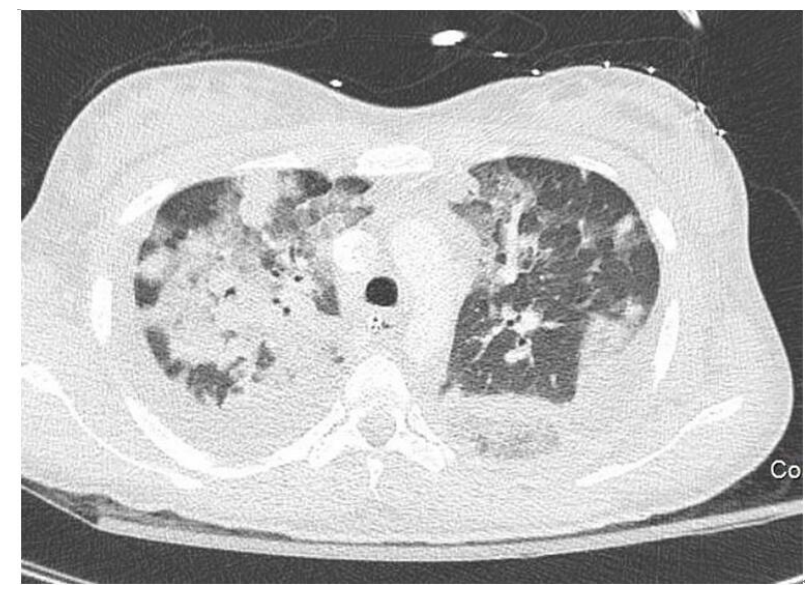

Figure 5. CT scan with diffuse pulmonary infiltrates with septic emboli and pleural effusions

Because of worsening hypoxic respiratory failure, the patient underwent endotracheal intubation. After respiratory 
stabilization, TEE showed both mitral and tricuspid valve vegetations (see Figure 2), a posterior mitral leaflet perforation (see Figure 3), and no evidence of PFO with the use of agitated saline. 3D TEE imaging was acquired as well, which aided in accurate measurement of vegetation dimensions (see Figure 4). Computed tomography of the chest, abdomen, and pelvis showed diffuse multifocal inflammatory infiltrates involving both lungs with a cavitary component, multiple wedge shaped hypodense lesions in the right kidney, and a wedge shaped hypodensity in the periphery of the spleen concerning for embolic infarcts (see Figure 5). She was taken to the operating room for surgical intervention shortly after intubation with concerns of worsening heart failure and valvulopathy.
Intraoperatively, a large perforation of the posterior mitral valve leaflet, an abscess in the posterior wall of the left atrium, and vegetations on the tricuspid and mitral valve were noted. Both the tricuspid and mitral valves were replaced and two permanent bipolar epicardial pacing wires were utilized. She was paced postoperatively and surface ECG three weeks after the operation showed a junctional rhythm without atrial activity (see Figure 6). An ECG using the permanent intracardiac leads showed atrial activity and complete heart block, which persisted for another month and required a permanent pacemaker at that time (see Figure 7). The patient was discharged to a rehabilitation facility with an extended course of antibiotics.

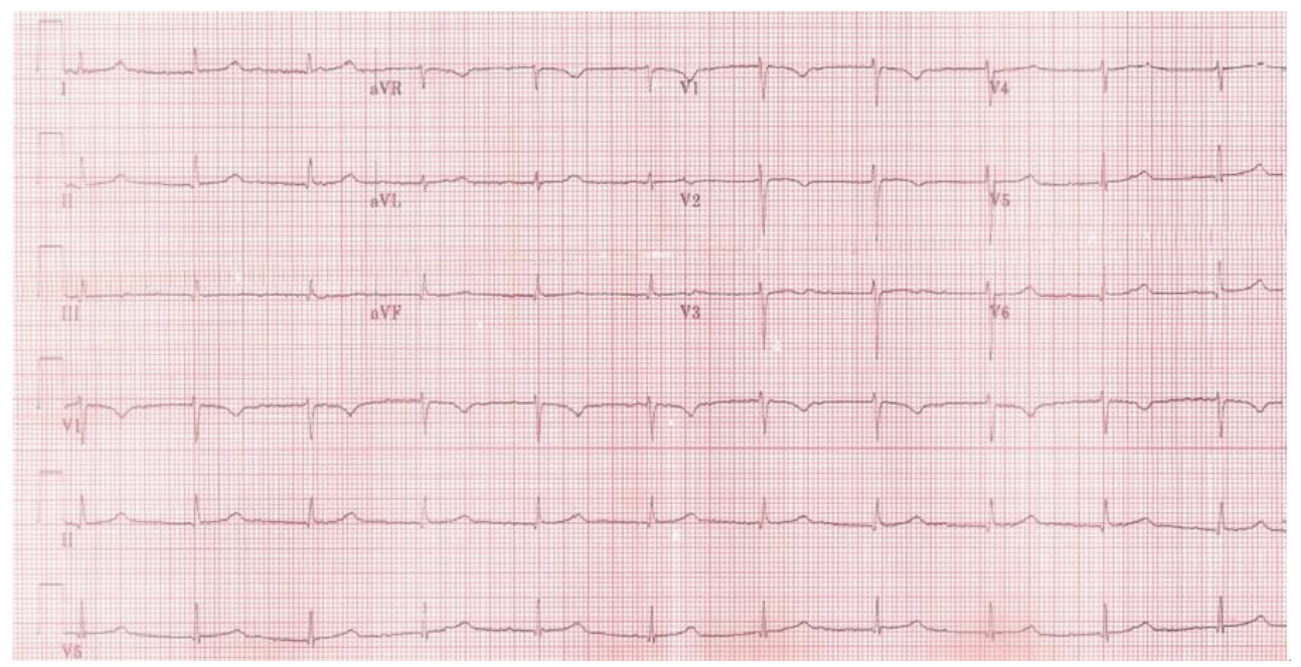

Figure 6. Post-operative surface ECG showing junctional rhythm

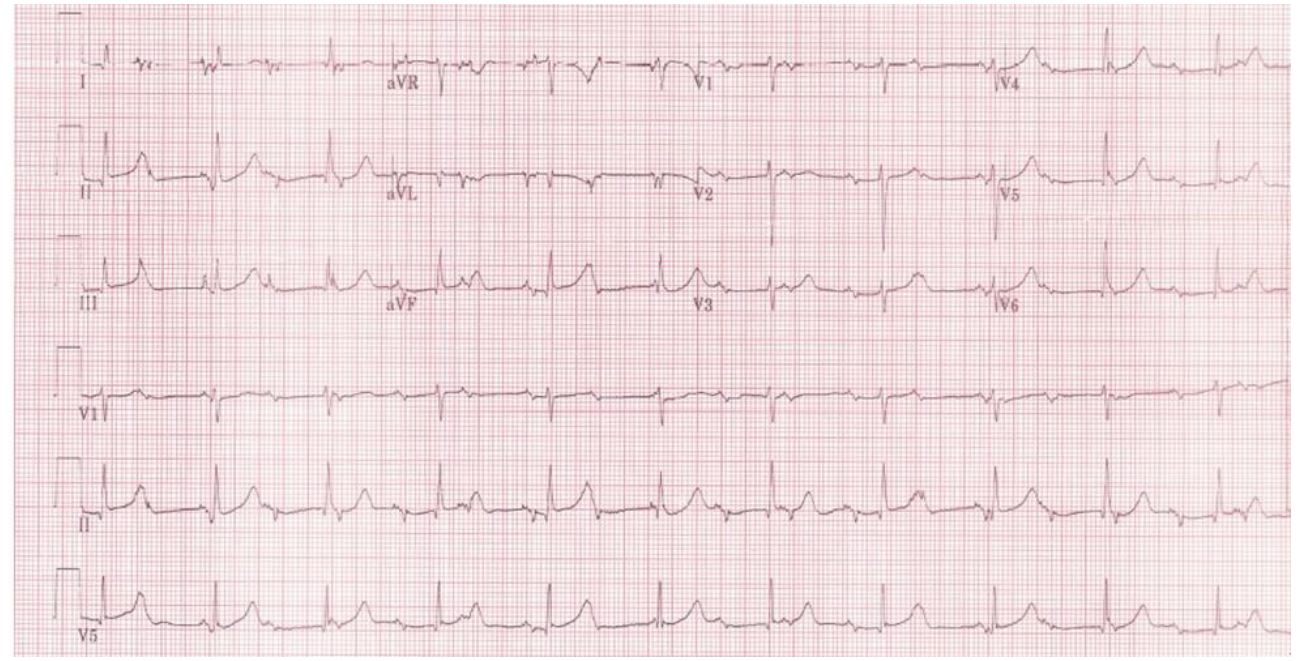

Figure 7. Post-operative intra-cardiac lead ECG showing atrial activity and complete heart block 


\section{Discussion}

This case highlights a unique presentation of IE and the importance and limitations of imaging to define valvular involvement. The patient presented with both tricuspid and mitral valve disease in the setting of IVDU which occurs in only $5 \%-10 \%$ of cases. ${ }^{[3]}$ After initial evaluation, the patient developed heart failure, necessitating surgical intervention. Heart failure has the greatest effect on prognosis and in most cases is due to worsening valvular regurgitation. ${ }^{[1]}$ The mitral valve perforation, identified on TEE using Doppler flow, contributed to her hemodynamic decline. Perforation or fistula formation can represent sequelae of uncontrolled infection from either periannular extension or abscess formation. ${ }^{[4]}$ TEE identified the perforation but failed to detect the mural abscess found intraoperatively showing the limitations of these imaging studies.

Systemic emboli occur in up to $50 \%$ of IE diagnoses. ${ }^{[1]}$ Recent studies have shown echocardiographic attributes of vegetations have a clear association with embolic risk including large vegetations $>10 \mathrm{~mm}$ and severe vegetation mobility. ${ }^{[5]}$ TEE characterized the size and mobility of the vegetations. In this case, computed tomography allowed for confirmation of multi-organ system emboli involving the spleen, kidneys, and lungs. Conduction abnormalities, namely AV block, are strongly associated with abscess formation and invasive infection though in this case are likely related to the surgical intervention required. ${ }^{[6]}$ While more common with aortic valve involvement due to anatomic proximity, AV block has been demonstrated with mitral and tricuspid valve infection as well, occurring up to $13 \%$ of cases. ${ }^{[6]}$ This case featured complete AV block requiring pacing with mitral and tricuspid valve involvement. Detection of this patient's conduction abnormality was initially difficult, as surface ECG showed junctional rhythm. Utilization of the permanent intracardiac leads with the 12-lead ECG revealed the accurate diagnosis of complete heart block.

Our case highlights the use of echocardiography (TTE, TEE, and 3D TEE) in the diagnosis of a unique case of endocarditis. The limitations of these modalities are evident in this case as well, given the intraoperative findings of a mural abscess that were not observed on initial echocardiography. The use of serial echocardiography for detection of extension of infection was precluded by the patient's rapid heart failure decompensation. Infective endocarditis can be a challenging diagnosis to make and multiple imaging modalities may be necessary.

\section{REFERENCES}

[1] Cahill TJ, Prendergast BD. Infective endocarditis. The Lancet. 2016; 387(10021): 882-893. PMid:20223755 http://dx.doi.org/10. 1093/ejechocard/jeq004

[2] Habib G, Badano L, Tribouilloy C, et al. Recommendations for the practice of echocardiography in infective endocarditis. European Journal of Echocardiography. 2010; 11(2): 202-19.

[3] Frontera JA, Gradon JD. Right-side endocarditis in injection drug users: review of proposed mechanisms of pathogenesis. Clin Infect Dis. 2000; 30(2): 374-9. PMid:10671344 http://dx.doi.org/1 $0.1086 / 313664$

[4] Anguera I, Jose M, Miro JM. Aorto-cavitary fistulous tract formation in infective endocarditis: clinical and echocardiographic features of 76 cases and risk factors for mortality. Eur Heart J. 2005; 26(3): 28897. PMid:15618052 http://dx.doi.org/10.1093/eurheartj /ehi034

[5] Thuny F, Disalvo G, Belliard O, et al. Risk of embolism and death in infective endocarditis: prognostic value of echocardiography: a prospective multicenter study. Circulation. 2005; 112(1): 6975. PMid:15983252 http://dx.doi.org/10.1161/CIRCULATI ONAHA . 104.493155

[6] Meine TJ, Nettles RE, Anderson DJ, et al. Cardiac conduction abnormalities in endocarditis defined by the Duke criteria. Am Heart J. 2001; 142(2): 280-5. PMid:11479467 http://dx.doi .org/10. $1067 / \mathrm{mhj} .2001 .116964$ 